\title{
DIAGNÓSTICO CITOPATOLÓGICO DE TUMORES NEUROEPITELIAIS PELA TÉCNICA DO ESFREGAÇO
}

\author{
LUIZ F. BLEGGI TORRES * - LUIZ MARTINS COLLACO **
}

\begin{abstract}
RESUMO - Estudamos grupo de 198 casos de tumores neuroepiteliais com diagnóstico peroperatório feito pela análise citológica de esfregaços, comparando seus indices de acuidade com o diagnóstico final em cortes de parafina. Èm 90,6\% dos casos, o diagnóstico final obtido na parafina foi similar ao feito em esfregaços. No grupo de casos em que o diagnóstico citológico não foi confirmado pelos cortes em parafina, na maioria dos casos não foi afetada a conduta neurocirúrgica imediata, representando diferenças em graduação de astrocitomas e gliomas mistos. Os critérios citológicos nos principais grupos de tumores são apresentados, junto com as dificuldades para a interpretação deste método valioso para diagnóstico per-operatório.
\end{abstract}

PALAVRAS-CHAVE: tumores neuroepiteliais, diagnóstico citopiatológico per-operatório, técnica do esfregaço.

Cytopathologie diagnosis of neuroepithelial tumours by the smear technique.

SUMMARY - We studied 198 cases of neuroepithelial tumours with intra-operative diagnosis made by smears comparing the accuracy rates with the final panaffin section diagnosis. In $90,6 \%$ of case the final diagnosis obtained on paraffin preparations was similar to that made on smears. In the group of cases with cytological diagnosis not confirmed by paraffin sections the majority of cases were unlike to affect immediate neurosurgical management, and represent mainly differences in grading of astrocytomas and mixed gliomas. The cytological diagnostic criteria are discussed together with the main difficulties for the interpretation of this valuabie approach to intra-operative neurosurgical diagnosis.

KEY WORDS: neuroepithelial tumours, intra-operative cytopathologic diagnosis, smear technique.

A utilização de esfregaços de tumores do sistema nervoso constitui excelente método diagnóstico per-operatório (biópsia de congelação), com elevados índices de acuidade diagnóstica 5,10,15,18. Esta técnica baseia-se na distensão de diminutos fragmentos teciduais entre duas lâminas, confeccionando-se película delgada de células. É utilizada em unidades neurocirúrgicas e neuropatológicas em diversos países 4,7,15. Nossa intenção é estimular colegas patologistas e neuro-oncologistas a adotarem este método diagnóstico, pela difusão dos principais pontos diagnósticos em cada tipo tumoral.

Trabalho realizado nas Unidades de Neuropatologia (*) e Citologia (**) dos Serviços de Anatomia Patológica do Hospital Nossa Senhora das Graças e Hospital de Clínicas da Universidade Federal do Paraná (UTPR): * Professor Adjunto, Ph.D. em Neuropatologia; ** Professor Auxiliar.

Dr. Luiz Flernando Bleggi Torres - Centro de Patologia de Curitiba - Rua Alcides Munhoa 433 - 80510 Curitiba PR - Brasil. 


\section{MATERIAL E MÉTODOS}

A técnica do esfregaço é utilizada como método diagnóstico per-operatório em nosso Serviço desde 1987. Durante esse periodo, um total de 307 espécimes neurocirúrgicos foram analisados em consulta per-operatória, exclusivamente por preparações citológicas pela técnica do esfregaço 18. Deste grupo, 198 casos $(64,5 \%)$ representaram tumores de tecido neuroepitelial, de acordo com a nova classificaçao de tumores do sistema nervoso da Organização Mundial da Saúde 19. Este casos foram todos analisados pela técnica do esfregaço, que consiste na compressåo de diminutos fragmentos teciduais, ao redor de 1 a $2 \mathrm{~mm} 3$, entre duas lâminas com pressăo suficiente para, ao puxarmos a lâmina superior, configurar-se na lâmina de baixo delicada pelfcula celular. Peło menos dois esfregaços de cada caso foram analisados.

Após a confecção dosi esfregaços, estes são fixados em álcool absoluto por 30 segundos (2 trocas), lavados em água corrente por 10 segundos, corados em azul de toluidina a $1 \%$ aquoso (TOL.) por 30 segundos, lavados em água corrente, desidratados em álcool absoluto por 30 segundos (2 trocas). Após isto são clareados em xilol e montados em bálsamo. importante que as laminas sejam vigorosamente agitadas nas soluções fixativas e de lavagem, para remoção de resíduos líquidos inadequados. Após o diagnóstico per-operatório as lâminas são desmontadas, descoradas em álcool absoluto e recoradas pela técnica de Papanicolaou 1, para arquivo permanente. Em situações de dificuldade diagnóstica e pesquisa científica, utilizamos métodos imunocitoquímicos, pelä técnica da avidina/biotina-peroxidase (ABV/DAB)3 para proteína glial ácida fibrilar (GFAP), proteína S-100, enolase neurônio específica, neurofilamento e vimentina (Dakopatts).

\section{RESULTADOS E CRITERIOS DIAGNOSTICOS}

Os critérios utilizados para diagnóstico de neoplasias neurogliais do sistema nervoso por preparações citológicas estão bem estabelecidos 2,4,11,16,18. Comentaremos pontos mais relevantes a algumas sugestões derivadas de nossa experiência pessoal 16.

A. Tecido Encefálico Normal - Aspecto citológico variado, conforme sua origem topográfica. Amostras telencefálicas corticais (Fig. 1). demonstram neurônios com formato piramidal, núcleos vesiculosos com nucléolos proeminentes. Astrócitos são células com núcleos ovalados e discretamente vesiculosos, cromatina delicada e clara; oligodendrócitos têm aspecto linfocitóide, cromatina densa e são menores que astrócitos. Micróglia é representada por células alongadas e bastonetóides, sendo raras em tecido normal e geralmente indicando situações reativas infecciosas do sistema nervoso. Vasos sanguíneos capilares têm aspecto filiforme delicado, endotélio uniforme. Vasos fibro-musculares possuem diâmetros maiores, porém com endotélio delicado e sendo visíveis os núcleos de células da camada muscular, dispostos transversalmente ao eixo iongitudinal vascular. Todos estes elementos citológicos estão distribuídos aleatoriamente, de maneira que a celuridade é baixa, polimórfica e harmônica. Em amostras da substáncia branca predominam oligodendrócitos e vasos fibro-musculares. Amostras cerebelares demonstram células de: Purkinje similares aos neurônios piramidais, porém observando-se ainda neurônios da camada granular, que apresentam citoplasma escasso, núcleos redondos com cromatina densa, regular e uniforme. Demais células com características similares às descritas anteriormente.

B. Astrocitoma ( $\mathrm{n}=44)$ - Demonstram tufos de células neoṕásicas, geralmente envolvendo vasos sanguineos. Esta relação com vasos deriva do íntimo contacto de pés astrocitários normais à parede vascular na barreira hêmato-encefálica. Estes vasos são pouco proliferados e mantêm, por vezes, aspecto próximo do normal. A celularidade do tumor é baixa e monótona, composta predominantemente de astrócitos com discreto pleomorfismo. Os núcleos são vesiculosos, ovalados ou reniformes com cromatina frouxa e salpicada, sem mitoses (Fig. 2). A carioteca apresenta focos de espessamento e indentaçóes. Há fundo fibrilar delicado, metacromático ao azul de toluidina e refringente, se observado sob luz pollarizada (Fig. 3). Este fundo é intensamente positivo para proteína glial ácida fibrilar por métodos imunocitoquímicos 1, representando o cltoplasma astrocitário rico neste filamento intermediário (F'ig. 4). Algunsi casos podem suscitar dúvidas diagnósticas, com estados de gliose reacional em que notamos, porém, proliferação astrocitáría normal e não neoplásica, com astrócitos tendo núcleos regulares e citoplasma estrelado, misturados a outros tipos celulares normais como oligodendroglia e neurônios, o que não é visto em situações neoplásicas. Tais focosı de gliose são comuns na periferia de infartos, abscessos ou craniofaringiomas e merecem especial cuidado diagnóstico para sua distinção de tumores neurogliais, sendo recomendado correlação clínico-tomográfica para melhor interpretação. 15.

C. Astacitoma Anaplásico (n-19) - Gliobastoma (n-64) - Săo neoplagias astrocitárias malignas que apresentam aspectos citológicos similares. Observam-se vasos sanguíneos prolife- 

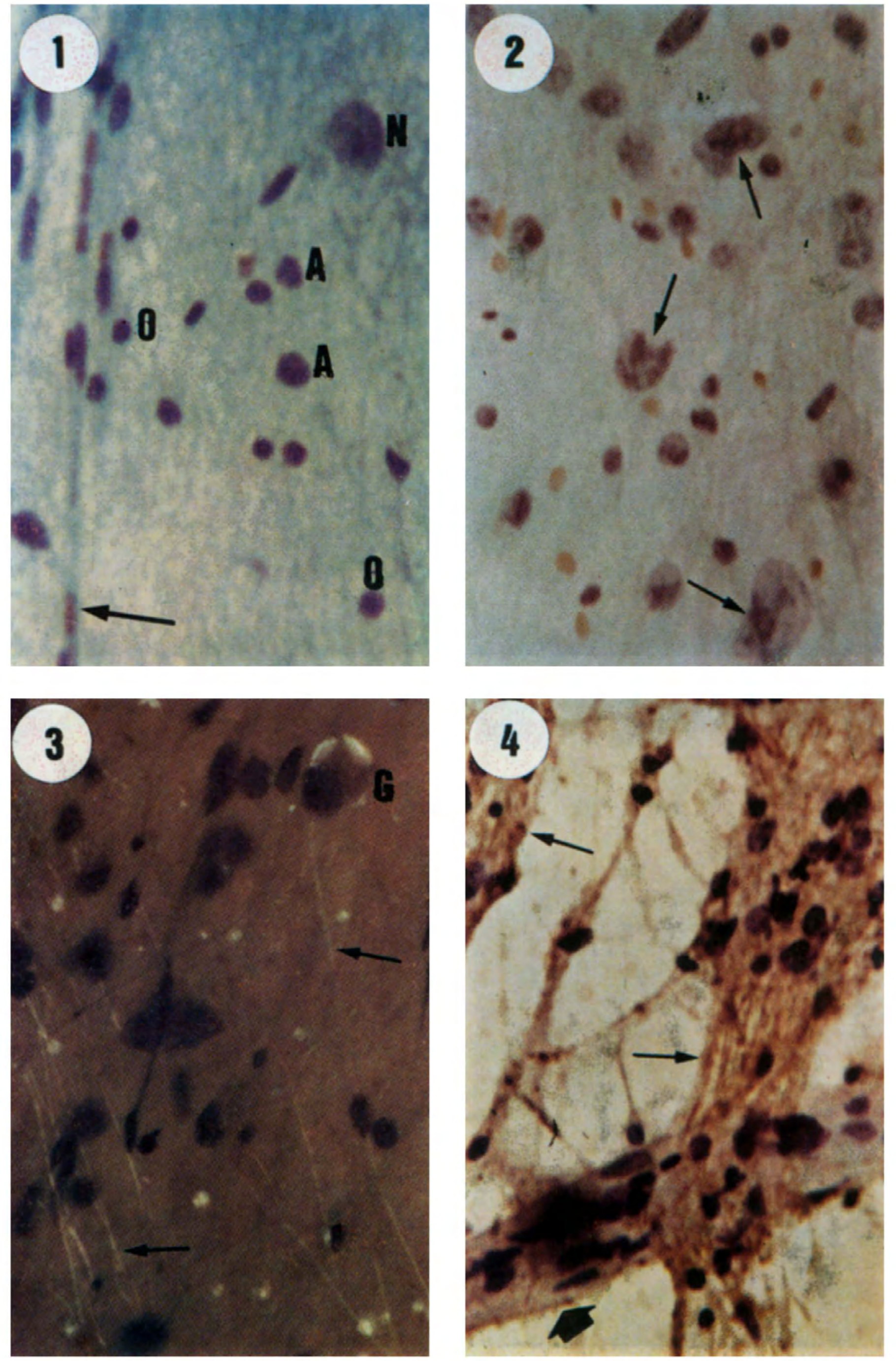

H'ig. 1. Esfregaco de tecido cerebral normal com vasos capilares (seta), celularidade variada $e$ polimorfa: neurónios $(N)$, astrócitos $(A)$ e oligodendrócitos $(O)$. TOL, $\times 400$.

Fig. 2. Astrocitoma com moderado pleomorfismo nuclear, carioteca indentada (setas), cromatina salpicada $e$ frouxa. Fundo fibrilar. Papanicolaou, $\times 400$.

Fig. 3. Astrocitoma anaplásico sob luz polarizada demonstrando refringencia delicada das fibrilas gliais metacromáticas (setas), bem como astrócito gemistocítico (G). TOL, $\times 400$.

Fig. 4. Imunocitoquimica para GFAP com positividade em citoplasma astrocitario $e$ fundo fibrilar, representados por precipitado acastanhado (seta fina). Vaso sanguineo negativo (seta grossa). AVB/DAB, $\times 400$. 

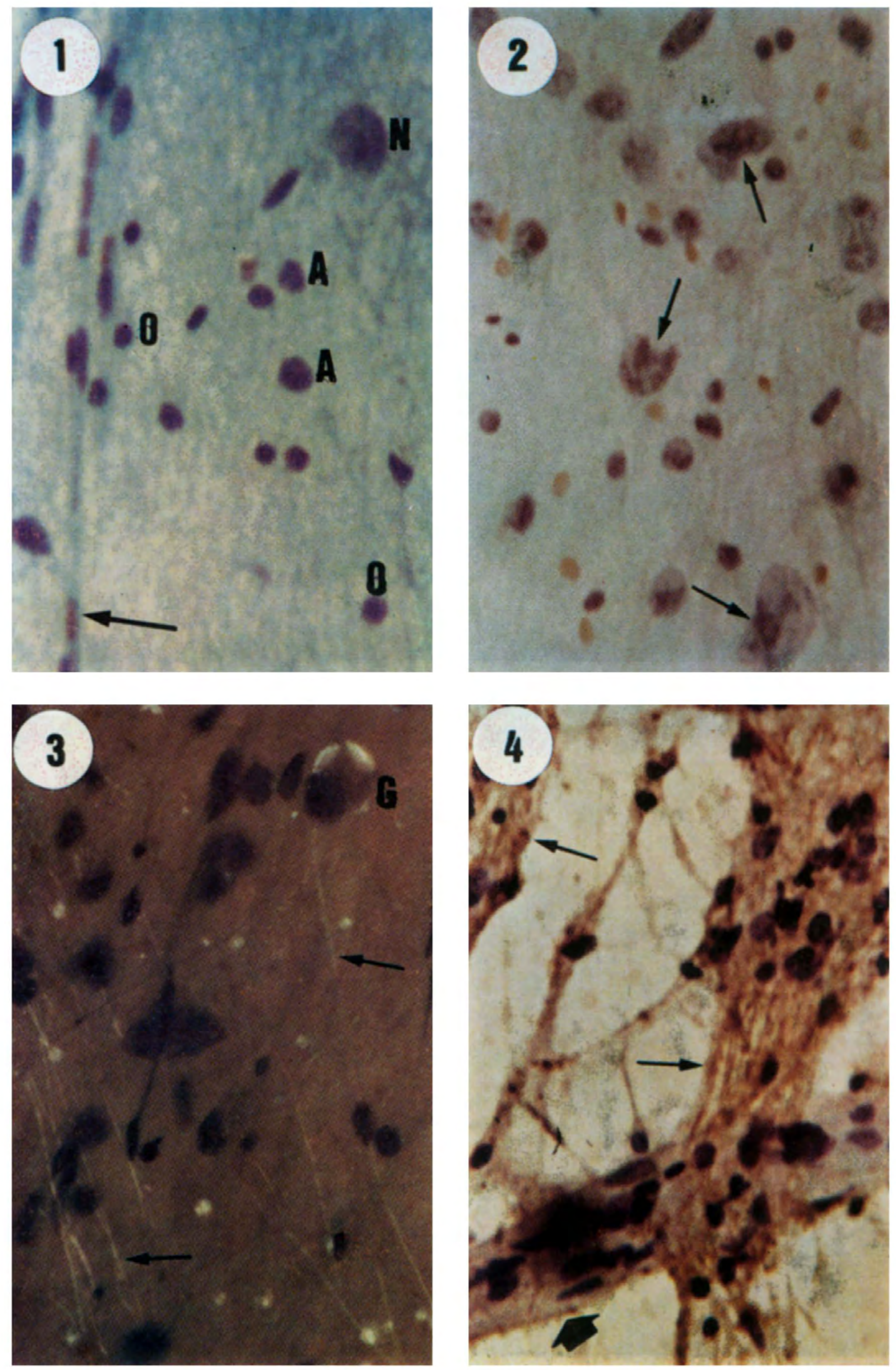

H'ig. 1. Esfregaco de tecido cerebral normal com vasos capilares (seta), celularidade variada $e$ polimorfa: neurónios $(N)$, astrócitos $(A)$ e oligodendrócitos $(O)$. TOL, $\times 400$.

Fig. 2. Astrocitoma com moderado pleomorfismo nuclear, carioteca indentada (setas), cromatina salpicada e frouxa. Fundo fibrilar. Papanicolaou, $\times 400$.

Fig. 3. Astrocitoma anaplásico sob luz polarizada demonstrando refringencia delicada das fibrilas gliais metacromáticas (setas), bem como astrócito gemistocitico $(G)$. TOL, $\times 400$.

Fig. 4. Imunocitoquimica para GFAP com positividade em citoplasma astrocitario $e$ fundo fibrilar, representados por precipitado acastanhado (seta fina). Vaso sanguineo negativo (seta grossa). AVB/DAB, $\times 400$. 
rados e com aspecto glomerulóide, apresentando endoteliose exsuberante, envoltos por tufos de células neoplásicas (Fig. 5). Esta proliferação vascular-endơtelial deriva da produçăo, pelas células neoplásicas, de fatores de proliferação de vasos, entre os quais o fator anglogenico tumoral, que estimulam o crescimento anormal da vascularização neoplásica nestas situaçðes de neoplasias astrocitárias malignas. 14. As células neoplásidas apresentam granđe anaplasia com cromatina grosseira e mitoses. Em casos de glioblastoma observa-se ainda presenca de focos de necrose tumoral, representados por restos celulares necrócitos e exsudato neutrofilico permeando o tumor (Fig. 6). Casos de glioblastoma de células gigantes demonstram, além dos elementos já mencionados, inúmeras células bizarras com cromatina grosseira e dimensర̃es enormes. Estes elementos gigantes parecem năo representar as células com malor atividade proliferativa e agressividade biológica, como mostram estudos com anticorpo Ki-67, sendo somente formas em regressão 9,14 .

D. Meduloblastoma $(n=20)$ - São tumores primitivos neuroectodérmicos originados da camada granular fetal cerebelar e que se caracterizam por afetar a fossa posterior de crianças e adultos jovens 18. Preparações citológicas demonstram lençol ricamente celular, composto por células monótonas e relativamente uniformes, citoplasma inaparente, distribuidas ao acaso e não relacionadas a vasos sanguíneos. Por vezes, observam-se cordões celulares ou esboço de estruturas rosetiformes. Os núcleos são hipercromáticos, redondos ou ovais, com carioteca espessa e octasionais mitoses. Focos de citólise individual também são geralmente vistos. Grande cuidado deve ser tomado para não confundir as células tumorais com neurónios granulares normais que, por vezes, podem vir com a amostra e estão geralmente misturados a outros elementos gliais e neuronais normais.

E. Ependimoma (n-12) - Demonstram íntima relação entre as células neoplásicas e vasos sanguíneos, com presença de inúmeras pseudo-rosetas perivasculares. As células ependimárias neoplásicas têm citoplasma fibrilar nadiado, núcleos uniformes, ovalados ou goticulares (Fig. 7). Cromatina regular e densa. Ocasionais mitoses e vasos sanguíneos cos. endotéllo pouco proliferado. Rosetas verdadeiras permitem o diagnóstico de certeza, porém são de dificil caracterizaçăo. Nas formas malignas os ependimomas apresentam maior celularidade com atipias nucleares, focos de necrose e proliferação vascular. Ependimomas mixo-papilares, encontrados geralmente na região de cauda eqüina, são compostos por eixo central hialino, amorfo e metacromático ao azul de toluidina, circundados por células ependimárias em arranjo radiado.

F. Oligodendroglioma $(n=6)$ - Neoplasias de difícil caracterização ao esfregaço, visto que apresentam lençóis celulares monótonos onde os limites citoplasmáticos são evidenciáveis dando, por vezes, aspecto similar ao visto em preparaçōes histológicas, com halo claro perinuclear. Os núcleos são redondos, regulares, com cromatina delicada e massas nucleolares discretas. Ocasionais mitoses. Carioteca delicada. Focos de calcificação por vezes presentes (Fig. 8). Em áreas, nota-se septaçăo dos ninhos celulares por trama vascular. Em casos malignos haverá necrose e proliferação vascular. Cuidado em separar esta entidade de ependimomas de células claras deve ser tomado 8 .

G. Demais lesões neuroepiteliais menos freqüentes - Neste grupo encontram-se casos de diagnóstico citopatológico mais difícil, devido a raridade e peculiaridade quanto a topografia e dados clínicos destas lesões. Astrocitomas subependimários de células gigantes: Afetam principalmente ventrículos laterais, determinando hidrocefalia e relacionam-se a esclerose tuberosa 14. Preparacões citológicas demonstram aspecto característico, representado por células grandes e piramidais com citoplasma amplo e núcleos ovalados ou vesiculosos, com nucléolos por vezes evidentes. Há intima relação com vasos sanguíneos, notando-se inúmeras pseudorosetas perivasculares. Estas células lembram neurônios e astrócitos gemistocíticos e parecem derivar de células gliais displásicas, com potencial de diferenciação ao longo de ambas as linhagens 14,18. Astrocitoma pilocítico: Origina-se em região hipotalámica e cerebelo de pacientes jovens. Em preparações citológicas se caracteriza por aspecto fibrilar difuso, núcleos discretamente alongados e com cromatina delicada, porém salpicada com focos discretos de reforço cariotecal. Ausência de mitoses. Especial cuidado deve ser dado em não exercer muita pressão na hora da confecção dos esfregaços; caso contrário, será induzido aspecto «pseudo-pilocítico» às células, as quais, porém, mostram compressão e estiramento nuclear, impossibłilitando análise adequada. Gliomas mistos: São reconhecidamente foco de dificuldade diagnóstica em preparações citológicas, porém não afetando a conduta neurocirúrgica imediata 18. Devem ser sempre lembrados em casos de oligodendrogliomas, pois estes tumores são, na maloria das vezes, gliomas mistos tipo oligo-astrocitomas. Papilomas de plexo coróide: Săo lesóes que accometem ventrículos laterais de crianças com eixo conjuntivo e vascular revestido por células epiteliais cúbicas, com citoplasma mediano e núcleos ovaís com cromatina delicada. Este aspecto assemelha-se muito ao de plexo corbide normal. devendo-se ter cuidado para conrirmar este 
diagnóstico. Im casos malignos há alteração na relaç̃o núcleo/citoplasma com atipias, mitoses, alteraçōes cromatínicas e necrose. Tumores embrionários, incluindo meduloepitelioma, neuroblastoma, ependimoblastoma e retinoblastoma. Afetam topografia especifica e têm aspecto citológico de neoplasia maligna de células pequenas, similar ao medulobastoma. Dados clínicos e correlacão tomográfica são indispensáveis à conclusão diagnóstica.

\section{COMENTARIOS}

O tratamento adequado de tumores neuroepiteliais depende de sua localização, tamanho e tipo histológico. Técnicas tomográficas e de ressonância magnética permi-tem altos indices de detecção desses tumores, porém o exame anátomo-patológico da peça neurocirúrgica ainda é mandatório para a definição do tipo histológico e consequente terapêutica complementar 11. A técnica do esfregaço com análise citopatológica. é importante adjuvante no diagnóstico de lesões obtidas por estereotaxia e representadas, na maioria das vezes, por pequenos fragmentos teciduais de difícil manuseio técnico por métodos histológicos convencionais. Além disto, a análise citopatológica de tumores fornece informaçóes valiosas e rápidas, com alto índice de acerto diagnóstico em consultas per-operatórias $2,11,15,17,18,20$.

$\mathrm{Na}$ presente série de casos de tumores de linhagem neuroepitelial tivemos concordância diagnóstica, entre a preparação citológica e o material de biópsia inclúído em parafina, em 90,4\% dos casos (Tabela 1). Entretanto, detectamos diferença diagnóstica citopatológica em 19 casos $(9,6 \%)$, porém sem implicação direta na conduta neurocirúrgica, visto que se tratavam de astrocitomas, com graduação histológica inadequada, devido à pouca representatividade do material recebido para esfregaço $(n=8)$, gliomas mistos nos quais só se valorizou um tipo citopatológico $(n=6)$ e tumores neuroepiteliais com tipo histológico erroneamente classificado ao esfregaço, porém com critério de malignidade corretamente estabelecido $(n=5)^{18}$.

Tabela 1. Diagnósticos citológicos de tumores neuroepiteliais confirmados pela parafina. Valores percentuais relacionados ao número total de casos estudados ( $n=307)$.

\begin{tabular}{lcr}
\hline Diagnóstico final & $\begin{array}{c}\text { Número de } \\
\text { ctasos }\end{array}$ & $\begin{array}{c}\text { Percentagem } \\
\text { geral }\end{array}$ \\
\hline Glioblastoma & 64 & 21,0 \\
Astrocitoma & 44 & 14,3 \\
Meduloblastoma & 20 & 6,5 \\
Astrocitoma anaplásico & 19 & 6,2 \\
Ependimoma & 12 & 4,0 \\
Oligodendroglioma & 6 & 2,0 \\
Glioma misto & 5 & 1,6 \\
Papiloma plexo coróide & 3 & 1,0 \\
Carcinoma plexo coróide & 2 & 0,6 \\
Pinealocitoma & 2 & 0,6 \\
PNET & 1 & 0,3 \\
Astrocitoma subependimário & 1 & 0,3 \\
Total & 179 & $58,3 \%$ \\
\hline
\end{tabular}

Outros autores detectaram indices de correlação semelhantes, oscilando de 87,5 a $95 \%$, incluindo além de tumores neuroepiteliais os tumores meníngeos e de nervo periférico 11,15. Entre os principais fatores de erro relatados estão a presença de processos inflamatórios atípicos, frequentes hoje em dia devido ao crescimento do número de pacientes com neuro-AIDS 6,12, bem como a pouca representatividade tumoral na amostra colhida 13 .

Finalmente, gostaríamos de entatizar que a utilização da técnica do esfregaço para analise per-operatória de neoplasias do sistema nervoso apresenta inúmeras vantagens, incluindo: (1) rapidez e confiabilidade; (2) ser adequada para espécimes pequenos obtidos em estereotaxia; (3) baixo custo operacional; (4) propiciar preparaçóes permanentes para arquivamento e para estudo imunocitoquimico complementar; 
(5) apresentar baixa incidência de falso positivo; (6) permitir ao patologista grande mobilidade, por não necessitar de micrótomo de congelação ou criostato para execução de consultas per-operatórias.

Agradecimentos - Os autores agradecem os demais patologistas e neurocirurgiסes de ambos os hospitais, pelo auxilio na coleta dos casos, bem como os técnicos e citotécnicos, pela confecção do material.

\section{R E E E R N I A S}

1. Bancroft J, Stevens A. Theory and Practice of Histological Techniques. Edinbourgh: Churchill Livingstone, 1982, p 332-363.

2. Bekerley BB, Adams JH, Doyle D, Graham DI, Harper CG. The smear technique in the diagnosis of neurosurgical biopsies. NZ Med J 1978, 87:12-15.

3. Bibbo M. Comprehensive Cytopathology. Philadelphia: W.B. Saunders, 1991

4. Burger PC. Use of cytological preparations in the frozen section diagnosis of central nervous system neoplasia. Am J Surg Pathol 1985, 9:344-354.

5. Cahill EM, Hidvegi DF. Crush preparations of lesions of the central nervous system: a useful adjunct to the frozen section. Acta Cytol 1985, 29:279-285.

6. Gray F, Gherardi R, Scaravilli F. The neuropathology of the acquired immune defictency syndrome (AIDS): a review. Brain 1988, 111:245-266.

7. Hume Adiams J, Grahan DI, Doyle D. Brain Biopsy: The Smear Technique for Neurosurgical Biopsies. London: Chapman and Hall, 1981, p 2-124.

8. Kawano $\mathrm{N}$, Yade $\mathrm{K}$, Aihara $\mathrm{M}$, Yashigita $\mathbf{S}$. Oligodendroglioma-like cells (clear cells) in ependymoma. Acta Neuropath (Berlin) 1983, 62:141-145.

9. Kepes JJ. Review of the WHO's new proposed classification of brain tumors. Annals of the XIth International Congress of Neuropathology, Kyoto, 1990, p 6.

10. Marshall LF. Adams JH, Doyle D, Graham DI. The histological biopsies. J Neurosurg $1973,39: 82-88$

11. Mouriquand C, Benabid AL, Breyton M. Stereotaxic cytology of brain tumours: review of an eight-year experience. Acta Cytol 1986, 31:756-764.

12. Navia BA, Petito CK, Gold JWM, Cho E-S, Jordan BD, Price RW. Cerebral toxoplasmosls complicating the acquired immune deficiency syndrome: clinical and neuropathological findings in 27 patients. Ann Neurol 1986, 19:224-238.

13. Ostertag CB, Mennel HD, Kiesling M. Stereotactic biopsy of brain tumours. Surg Neurol 1980, $14: 275-283$.

14. Russell DS, Rubinstein LJ. Pathology of Tumours of the Nervous System. Ed5. London: Edward Arnold, 1989.

15. Taratuto AL, Sevlever G, Piccardo P. Clues and pitfalls in stereotaxic biopsy of the central nervous system. Arch Pathol Lab Med 1991, 115:596-602.

16. Torres LFB. Citopatologia do sistema nervoso: critérios diagnósticos para análise de esfregaços de tumores do sistema nervoso. XIII Congresso Brasileiro de Citopatologia, 1991.

17. Torres LFB, Almeida $R$, Avila $S$, Alessi $S$, Freitas $R$. Brains tumours in South Brazil: a retrospective study of 438 cases. Arq Neuro-Psiquiat (São Paulo) 1990, 48:279-285.

18. Torres LFB, Collaço LM. Análise citológica per-operatória de lesóes do sistema nervoso central e periférico. Anais do XVIII Congresso Latino Americano de Patologia 1991, 1:101 (Abst).

19. World Health Organization. Histological Typing of Tumors of the Central Nervous System. Geneva: WHO, 1991.

2a Zhang YX, Luo KS, Liv JC, Chen Y, Chen YH, Lai RS. Cytological diagnosis of 500 cases of intracranial tumors during craniotomy. Chinese J Clin Cytol 1987, 3:19-27. 